\title{
Can the trace formula describe weak localisation?
}

\author{
R S Whitney, I V Lerner and R A Smith \\ School of Physics and Astronomy, University of Birmingham, Edgbaston, \\ Birmingham, B15 2TT, United Kingdom.
}

\begin{abstract}
We attempt to systematically derive perturbative quantum corrections to the Berry diagonal approximation of the two-level correlation function (TLCF) for chaotic systems. To this end, we develop a "weak diagonal approximation" based on a recent description of the first weak localisation correction to conductance in terms of the Gutzwiller trace formula. This semiclassical method is tested by using it to derive the weak localisation corrections to the TLCF for a semiclassically disordered system. Unfortunately the method is unable to correctly reproduce the "Hikami boxes" (the relatively small regions where classical paths are glued together by quantum processes). This results in the method failing to reproduce the well known weak localisation expansion. It so happens that for the first order correction it merely produces the wrong prefactor. However for the second order correction, it is unable to reproduce certain contributions, and leads to a result which is of a different form to the standard one.
\end{abstract}

\section{Introduction.}

Much recent research has concentrated on exploiting the similarities between quantum chaos (the quantum behaviour of systems which are classically chaotic) and disordered conductors in order to gain a better understanding of both [1-7] For both types of system one cannot calculate energy levels and eigenfunctions explicitly since these do not have simple analytic forms due to the non-integrability of the systems. One therefore concentrates on the statistical properties of the energy levels and wavefunctions. The key similarity between quantum chaotic and disordered systems is that, in an appropriate regime, their energy level spectra both have statistics described by random matrix theory (RMT) [8-10]

The techniques used to analyse the two systems (quantum chaotic and disordered conductors) are very different, and it is this which makes the similarities in the level statistics so potentially fruitful. Quantum chaos makes use of the Gutzwiller trace formula [11], a semiclassical relation in which all classical periodic orbits are summed over to obtain the quantum mechanical density of states. In most work a particular system is considered, and statistical averaging is performed over an energy window in the spectrum. In disordered conductors, the microscopic details of the disorder potential are neither known nor interesting, and this enables one to average over all realisation of disorder from the very beginning. This allows one to develop an effective field theory which has a well-defined perturbation expansion. As a consequence it is possible to calculate statistical quantities in disordered conductors in several important regimes. More specifically there are two important length scales in a disordered conductor: the system size, $L$ and the elastic transport mean free path, $\ell=v_{F} \tau$, where $\tau$ is the elastic transport scattering time, $v_{F}$ is the Fermi velocity. Using the 
diffusion constant $D=v_{F}^{2} \tau / d$, we can generate two time scales: $\tau$ and the ergodic time, $\tau_{\text {erg }}=L^{2} / D \sim(L / \ell)^{2} \tau$, which is the time taken for an electron to explore the whole sample. This leads to the following time regimes: the ergodic regime, $\tau_{\text {erg }}<t$, where the electron has explored the whole sample; the diffusive regime, $\tau_{\text {erg }}<t<\tau$, where the electron is diffusing through the sample as it scatters off impurities; and the ballistic regime, $t<\tau$, where the electron is moving ballistically between scatters. These of course lead to related energy regions via the uncertainty relation: ergodic, $E<E_{T h}$; diffusive, $E_{T h}<E<\hbar / \tau ;$ ballistic, $\hbar / \tau<E$, where the Thouless energy, $E_{T h}=\hbar / t_{\text {erg }}=\hbar D / L^{2}$. Finally, there is the energy scale $\Delta$, the mean energy level spacing which defines a further energy regime within the ergodic regime: the quantum regime, $E<\Delta$, corresponding to times longer than the Heisenberg time, $t>t_{\mathrm{H}}=\hbar / \Delta$. In the disordered conductor one can calculate the level statistics in all these regimes, $[8,12,13]$ and it shows RMT behaviour in the ergodic regime. In contrast, the Gutzwiller approach has so far been consistently applied within the diagonal approximation developed by Berry [14], although efforts have been made to go beyond this approximation by considering action correlations[15]. In the diagonal approximation, the leading contribution to the two-level correlation function (TLCF) has been found in the ergodic regime of quantum chaotic systems [14], and in the ergodic and diffusive regime of disordered systems[1]. Unfortunately, this method only works outside the quantum regime; in particular, it does not allow one to calculate the corrections in powers of $t / t_{H}$ predicted by RMT in the ergodic regime for systems with time-reversal invariance. This might lead one to ask the following questions: (i) Are the level statistics in quantum chaotic systems still described by RMT within the quantum regime? In particular, do the corrections in powers of $t / t_{H}$ predicted by RMT exist in the ergodic regime? (ii) Are there any analogues of the diffusive or ballistic regimes in quantum chaotic systems?

It is the possibility of answering such questions as these that motivates work into the analogies between chaotic and disordered systems.

One approach to answering such questions would be to develop a powerful field theoretical technique for quantum chaotic systems analogous to that which exists for disordered metals $[16,17]$. This field theory should be able to reproduce Berry's result as the first term in a perturbation expansion. An effective field theory - the "ballistic" sigma-model [2-4] - has recently been developed for quantum chaos. The only averaging used was over an energy window in the spectrum, and this seemed sufficient to reproduce RMT behaviour. However, this method lacks a well-defined perturbation expansion, unless one introduces additional statistical averaging over some ensemble [18-20]

The aim of this paper is to establish whether a regular perturbative expansion can be formulated within semiclassical methods based on the Gutzwiller trace formula. In particular, we will concentrate on the two-level correlation function (TLCF), $R(\omega)$, and its Fourier transform, the spectral form factor (SFF), $K(t)$. We consider the model of randomly distributed semiclassical scatterers $[5,6]$. Standard scaling considerations [21] ensure that for sufficiently large systems the results should coincide with those derived for a system with Gaussian white-noise disorder [12, 22], which is the conventional model for diagrammatic considerations. Note that in the diffusive regime in two dimensions the one loop result (corresponding to the diagonal approximation) vanishes, and the two loop result is the leading contribution [22] for systems with time-reversal invariance, whilst the three loop result is the leading contribution for systems without time-reversal invariance. 
Verifying such an approach for disordered systems should be the first step before one can apply the same methods to generic quantum chaotic systems. Perturbation theory is used to describe the diffusive regime, whose existence in the disordered system is due to the separation of the time scales, $\tau$ and $t_{\mathrm{erg}}$, by the large parameter $(L / \ell)^{2}$. Such a parameter does not exist in a generic chaotic system. Nevertheless, numerically $t_{\text {erg }}$ is always much longer than the 'ballistic' time, $\tau$. It might be that the perturbation expansion of $K(t)$ for $\tau<t<t_{\text {erg }}$ would reveal behaviour which is more universal than that characterised just by the contribution from short orbits. Perturbation theory is also used to describe the ergodic regime of disordered systems. It is known from the exact solution [8] that there are correction in powers of $t / t_{H}$ to the leading order diagonal contribution to the SFF (which is linear in $t$ ). For such systems, perturbation theory is able to reproduce these corrections. However in the case of a generic chaotic system, it is not known whether such corrections necessarily exist; if perturbation theory could be applied to these systems this could be checked.

Therefore, we will try to develop a regular perturbation expansion based on the Gutzwiller approach. In this case, the SFF is contributed by the sum over all pairs of closed orbits which particles traverse in the system. The diagonal approximation [14] suggests that only identical (or time-reversed, if allowed) paths make a contribution which does not disappear after averaging over some energy interval. The reasoning behind this is that different orbits have uncorrelated classical actions, and hence their contributions to the SFF have uncorrelated phases and average to zero.

Perturbation theory for $K(t)$ in the disordered metal [12, 1] gives not only the diagonal result in leading order, but also weak localisation corrections [22] at higher order. Recently we have shown[23] how to interpret the weak localisation corrections for disordered metals in terms of a trajectory picture. It is tempting to use this trajectory picture for actual calculations in the language of the trace formula.

In the trajectory picture, we identified paths contributing to the SFF which are identical for most of their length, except at regions where a path self-intersects. At these regions it is possible to make two different paths by connecting up the partial paths in different ways. We thus obtain a set of contributions to the SFF where the actions corresponding to the two paths are almost identical, whilst the paths themselves are piecewise identical (see Fig. 1). We introduce the terminology weak diagonal approximation to describe such paths in contrast to Berry's strong diagonal approximation. The perturbative order of a given contribution is the number of loops created by self-intersections (so that the strong diagonal approximation is the one-loop term). In disordered metals, the regions where the paths are identical correspond to diffusion propagators; the self-intersection regions to the Hikami boxes [24, 17]. The latter are regions with size of order $\ell$ where $s$-wave scattering ensures the gluing together of diffusive paths. In a general chaotic system, the loops are made by gluing together (by diffraction, tunneling or some other quantum process) classical trajectories to make a closed orbit. By analogy with the disordered metal, we call such a gluing region a Hikami box. We believe that the whole question of whether a semiclassical approach can reproduce perturbation theory will reduce to the question of whether it can correctly obtain the Hikami boxes - they are the glue which holds the coherent partial paths together.

To carry out the test of semiclassical theory proposed in the last paragraph, we need to use a particular semiclassical approach and work up to a particular order of perturbation theory. A number of semiclassical methods exist for dealing with what we call the Hikami boxes [25-27,5] However since these only calculate the leading 
order weak localisation correction to conductivity in disordered metals, they deal with a relatively simple Hikami box. To test the validity of a semiclassical approach one should check whether it reproduces the higher order Hikami boxes. We take the approach based on the Gutzwiller trace formula, developed by Argaman[5], as such an approach would have a good chance of being directly applicable to chaotic systems. We calculate the SFF, and hence the TLCF, to third loop order in the perturbation expansion (which involves the calculation of higher order Hikami boxes). Note that the two (three) loop order gives the leading contribution to the TLCF in the diffusive regime of a disordered system with (without) time-reversal symmetry. Unfortunately, this semiclassical method gives the correct functional form only up to two loop order. Beyond this, it does not work. In particular, we show that it can not reproduce the three-loop result. It appears that this method, although quite general and very attractive because of its simplicity and obvious physical interpretation, cannot correctly obtain the Hikami boxes. This does not mean that the Hikami boxes cannot be reproduced by semiclassical methods: it has been shown [6] that this is possible for exactly the system we consider in this paper. However, it is not clear whether the technique suggested in [6] may be extended to generic chaotic systems. Since the method we consider here is based on the Gutzwiller formula, if it were successful, it would be completely general.

The rest of the paper is organised as follows. In section 2, we discuss the weak diagonal approximation and the regimes in which it will apply. In section 3, we briefly review the derivation, from the Gutzwiller trace formula, of a semiclassical expression for the SFF, and the application of the strong diagonal approximation to this expression for the SFF. We then construct the weak diagonal expansion in terms of this semiclassical expression for the SFF. In sections 4 and 5 we calculate the twoloop and three-loop contributions to the SFF (the first and second terms in the weak diagonal expansion) respectively. In sections 6 and 7 we use the results of sections 4 and 5 to write explicit formulas for the leading contributions to the TLCF of a twodimensional disordered system in the diffusive regime with and without time-reversal symmetry. In section 8 we consider whether the techniques used for the diffusive regime can be extended to the ergodic regime. Finally in section 9 we discuss our results.

\section{The Weak Diagonal Approximation.}

In this section we explain in more detail what is meant by the weak diagonal approximation. We then discuss the timescales for which such an approximation would be valid.

The weak diagonal approximation involves evaluating the contribution of paths that are nearly-identical. In this context nearly-identical means the pair of paths follow each other everywhere, except when the paths come close to themselves. In such regions ("Hikami boxes" marked by dashed boxes in Fig. 1) the two paths differ: one path crosses in this region, while the other does not. Two paths are considered close when their separation in phase space satisfies $\delta r \delta p \leq \hbar$. If the system has time-reversal symmetry then $\delta p$ is either the sum or the difference of the momenta.

We consider systems in which the Hikami boxes are due to quantum mechanical effects such as diffraction, tunnelling, etc. The time scale, $t_{Q}$, of such quantum scattering events, will be infinite in the classical limit $\hbar \rightarrow 0$, and much larger than the flight time (mean free time) $\tau$ in semiclassical (disordered) systems. Nonetheless, 
(a)
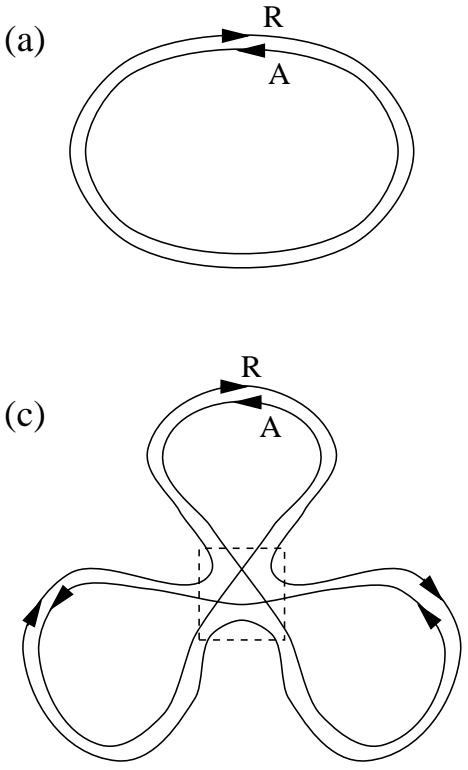

(b)

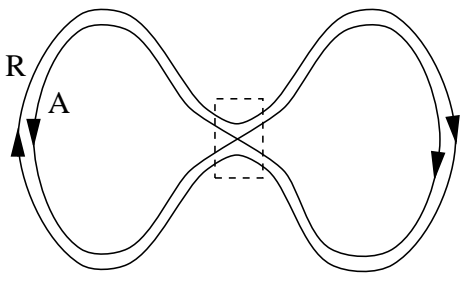

(d)

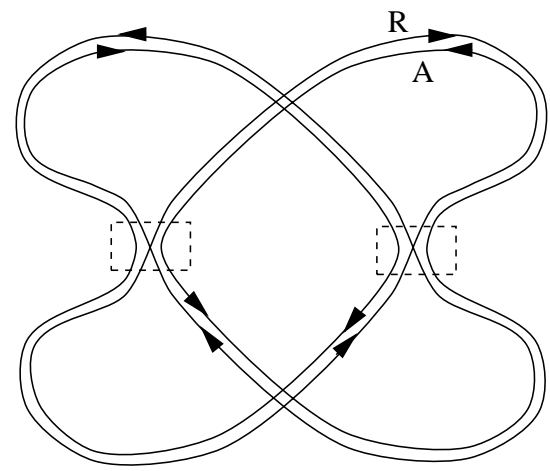

Figure 1. A schematic representation of the first few contributions to the weak diagonal approximation of the TLCF. The two paths in each figure follow each other exactly, except in the dashed boxes. In the dashed boxes, which correspond to Hikami boxes, quantum scattering causes one path to cross while the other does not. In (a) the paths are identical everywhere, this gives $R_{1}(\omega)$ which equals the strong diagonal approximation. The geometry of paths contributing to $R_{2}(\omega)$ are shown in (b). The two different geometries of paths contributing to $R_{3}(\omega)$ in a system without time reversal symmetry, are shown in (c) and (d).

The arrows on the retarded Green's functions ( $R$ ) point in the direction of the underlying path, while those on the advanced Green's functions (A) point in the opposite direction.

these quantum events are of great importance for any paths with a period larger than $t_{Q}$. These quantum scattering events cause a particle to be spread among a bunch of classical paths which are close in phase space.

For paths with a period much larger than $t_{Q}$, the contribution of a given pair of nearly-identical paths will be of the same order as the contribution of identical paths. However the probability of a path coming close to itself in phase-space in time $t$ is small if $t \ll t_{\mathrm{H}}$, and hence the weak diagonal approximation provides perturbative corrections to the strong diagonal approximation. The strong diagonal approximation arises from a path which forms a single loop (Fig. 1a). The leading order correction comes from the paths with one self-intersection which form two loops, as shown in Fig. 1b. The next order correction comes from the paths with two self-intersection which form three loops (e.g. Fig. 1c,d) and so on. This loop expansion is totally equivalent to the standard field-theoretical loop expansion[23].

In the ergodic regime, $t_{\mathrm{erg}} \ll t \ll t_{\mathrm{H}}$, the probability of a path coming close to itself is of order $t / t_{\mathrm{H}}$. Here one expects universal behaviour which is described by RMT. The strong diagonal approximation reproduces only the linear behaviour of the form factor $K(t)$. The weak diagonal approximation allows the calculation of perturbative corrections in $t / t_{\mathrm{H}}$, thus raising the possibility of checking whether 
chaotic systems fully obey RMT $[8,9]$ in this regime. Note that the very possibility of this expansion depends on whether the condition $t_{Q} \ll t_{\mathrm{H}}$ is satisfied. This is not necessarily satisfied for a generic billiard of typical size $L$, for which $t_{Q} \sim \tau\left(L / \lambda_{E}\right)^{\alpha}$, where the flight time $\tau \propto L$, and $\lambda_{E}$ is the De Broglie wavelength of a particle with energy $E$. As $t_{\mathrm{H}} \propto L^{2}$ for a two-dimensional billiard, the above inequality is parametrically valid only for $\alpha<1$.

We have chosen to apply the weak the weak diagonal approximation to a system of randomly placed semiclassical scatterers because the above conditions are easily satisfied. In this case, the quantum scatterings are mainly due to the paths grazing the scatterers, so the theory of penumbra diffraction [28] can be applied. Then for a system with $N$ scatterers of radius $R$ per unit volume $t_{Q} \sim \tau\left(R / \lambda_{E}\right)^{2 / 3}$, where $\tau=\left(v_{E} N R^{d-1}\right)^{-1}$. This guarantees that $t_{Q} \ll t_{\mathrm{H}}$ even if the system size $L$ is of order $R$. If we consider an extended system of size $L \gg v_{E} \tau$, then $t_{Q} \ll t_{\text {erg }}$, and the weak diagonal approximation can be applied to the diffusive regime of this system. In this regime the probability of self-intersection is parametrically smaller than $t / t_{\mathrm{H}}$. The quantum parts of trajectories (Hikami boxes) are the same as in the ergodic regime whilst the classical parts give a different contribution as they are too short to explore all the phase space. When applied to the diffusive regime of a system with a Gaussian white-noise disorder potential, the weak diagonal approximation still reproduces [23] the standard weak localisation corrections [17, 12]. The system of semiclassical scatterers in the limit of $L \gg R$ must have weak localisation corrections of the same form as those for the white-noise disorder; therefore, any consistent semiclassical method must reproduce these results.

It is interesting to ask whether an analogous non-ergodic regime exists for generic chaotic systems. For this the time-scales must be arranged as $\tau \ll t_{Q} \ll t_{\text {erg. }}$. Since $\tau$ and $t_{\text {erg }}$ are parametrically the same, the numerical window between them should be large enough to squeeze $t_{Q}$ inside. It remains to be seen whether such a condition may be satisfied by any billiard.

The final timescale we should consider is the Erhenfest time $t_{\mathrm{Erh}}=\lambda^{-1} \ln \left(R / \lambda_{E}\right)$, where $\lambda$ in the Lyapunov exponent [6]. For a system of semiclassical scatterers it is clear that $t_{\text {Erh }} \ll t_{Q}$ For a generic billiard, $t_{\text {Erh }}$ will depend on the logarithm of $\left(L / \lambda_{E}\right)$, while it seems likely $t_{Q}$ will depend on some power of $\left(L / \lambda_{E}\right)$. In the semiclassical limit, $\lambda_{E} \ll R$, we expect that such a system will also have $\tau \ll t_{\text {Erh }} \ll t_{\mathrm{Q}}$. This will be of relevance to the details of how we construct the Hikami boxes in the next section.

\section{A semiclassical description of the strong and weak diagonal approximations.}

The two level correlation function (TLCF) is given by,

$$
R(\omega)=\frac{1}{\nu^{2}}\left\langle\nu\left(E+\frac{1}{2} \omega\right) \nu\left(E-\frac{1}{2} \omega\right)\right\rangle-1
$$

where $\nu(E)$ is the density of states per unit volume. Given the spectrum of the system, $\left\{E_{n}\right\}, \nu(E)$ can be written as,

$$
\nu(E)=\frac{1}{L^{d}} \sum_{n} \delta\left(E-E_{n}\right)
$$


and the average density of states, $\nu=\langle\nu(E)\rangle=\left(\Delta L^{d}\right)^{-1}$. The averaging denoted by $\langle\cdots\rangle$ is carried out over a certain energy window in the spectrum of the chaotic system's spectrum. In disordered systems it is also possible to average over the ensemble of all realisations of the disorder. Ensemble averaging is used in the vast majority of work on disordered systems, because it is simpler and better understood. In both chaotic and disordered systems, the mean level spacing is given by the Weyl rule, so a system of volume, $L^{d}$, at an energy, $E$, has $\Delta=h^{d} / \Omega_{E}$. The volume of the phase space constant energy surface is $\Omega_{E}=\left(S_{d} p_{E}^{d-1} L^{d}\right) / v_{E}$, where $S_{d}$ is the surface area of a $d$-dimensional sphere of unit radius, and $p_{E}$ and $v_{E}$ are the momentum and velocity of a particle with energy, $E$.

We consider a system of non-interacting electrons in a potential which varies slowly on the scale of the wavelength of the electrons at the Fermi surface. In this semiclassical limit, the density of states is given by the Gutzwiller trace formula [11],

$\nu(E)-\nu=\sum_{\alpha}\left(A_{\alpha}(E) \exp \left[i S_{\alpha}(E) / \hbar\right]-A_{\alpha}^{*}(E) \exp \left[-i S_{\alpha}(E) / \hbar\right]\right)$

where the summation is over all periodic classical paths, $A_{\alpha}(E)$ and $S_{\alpha}(E)$ are the amplitude and action of the $\alpha$ th classical path. For convenience we have defined $A_{\alpha}(E)$ so that it includes the phase factor due to the Maslov index. When studying spectral statistics semiclassically, it is often more convenient to consider the spectral form factor (SFF), $K(t)$, than the TLCF itself,

$$
K(t)=\left\langle\sum_{\alpha, \beta} A_{\alpha} A_{\beta}^{*} \exp \left[i\left(S_{\alpha}-S_{\beta}\right) / \hbar\right] \delta\left[t-\frac{1}{2}\left(T_{\alpha}+T_{\beta}\right)\right]\right\rangle
$$

where the period of the classical periodic path, $\alpha$, is $T_{\alpha}=\mathrm{d} S_{\alpha}(E) / \mathrm{d} E$. The TLCF is related to the $\mathrm{SFF}$ in the limit that $\omega \ll E$, by

$$
R(\omega)=4 \Delta^{2} \Re \mathrm{e}\left[\int_{o}^{\infty} \mathrm{d} t \exp [i \omega t / \hbar] K(t)\right]
$$

Berry introduced the diagonal approximation of (4) [14], arguing that after the averaging was carried out, only terms where $\alpha=\beta$ would still contribute to the summation. All terms with $S_{\alpha} \neq S_{\beta}$ oscillate wildly as the averaging is carried out, and so these terms will be negligible. Berry's diagonal approximation assumes that those terms for which $\alpha \neq \beta$ but $S_{\alpha}=S_{\beta}$ are rare enough that one can ignore them. We will call this approximation the strong diagonal approximation, to distinguish it from the weak diagonal approximation that we will introduce below. When applied to the ergodic regime in a generic chaotic system, the strong diagonal approximation was found to reproduce the leading order behaviour of the spectral form factor in random matrix theory. More generally, it was shown that the contribution of these strong diagonal terms to the SFF is given by [1],

$$
K_{1}(t)=\frac{2}{\beta} \frac{t}{(2 \pi \hbar)^{2}} P(t)
$$

where $\beta=1$ if the system has time reversal symmetry, and $\beta=2$ if it does not. Here $P(t)$ is the probability of returning to the same point in phase space, in a time, $t$, integrated over the phase space surface of constant energy, $E$. We now introduce notation that will be of use later on, let $f_{E}\left(\boldsymbol{r}^{\prime}, \boldsymbol{p}^{\prime}, t ; \boldsymbol{r}, \boldsymbol{p}\right)$ be the probability that a 
particle which is initially at $(\boldsymbol{r}, \boldsymbol{p})$, is at $\left(\boldsymbol{r}^{\prime}, \boldsymbol{p}^{\prime}\right)$ after a time, $t$, given that the particle's final energy, $E$, is constrained to equal its initial energy. Then (6) becomes

$$
K_{1}(t)=\frac{2}{\beta} \frac{t}{(2 \pi \hbar)^{2}} \int \mathrm{d} \boldsymbol{r} \mathrm{d} \boldsymbol{p}_{E} f_{E}(\boldsymbol{r}, \boldsymbol{p}, t ; \boldsymbol{r}, \boldsymbol{p})
$$

where $\mathrm{d} \boldsymbol{p}_{E}=\mathrm{d} \boldsymbol{p} \delta[H(\boldsymbol{r}, \boldsymbol{p})-E]$, in other words the integral is restricted to the surface in phase space with energy, $E$. When applied to the diffusive regime of a twodimensional disordered system, this result is $t$-independent, which means it gives no contribution to the TLCF.

We now attempt to develop the weak diagonal approximation for a semiclassical system based on the Gutzwiller Trace formula. To do this we must consider how to deal with the regions where the paths are not identical (Hikami boxes). A path $\alpha$ that comes close to itself, will typically stay close to itself for a time of the order of the Erhenfest time $t_{\text {Erh }}$. Since $t_{\text {Erh }} \ll t_{\mathrm{Q}}$, the nearest quantum scatterer on the path will be some distance from the region where the path comes close to itself. Therefore the behaviour of path $\alpha$ in the Hikami box can be considered as classical. Path $\beta$ is the path that will cross in the Hikami box, it will be identical to $\alpha$ up to the last quantum scattering before the region in which $\alpha$ is close to itself. Path $\beta$ must leave that scatterer with a slightly different momentum from path $\alpha$, and so moves away from it slowly before converging slowly towards the other leg of path $\alpha$. Finally $\beta$ becomes close enough to $\alpha$ that at the next quantum scattering the two paths become identical again. When path $\alpha$ comes close to itself, the distance between the two parts of the path is of order of a wavelength, $\lambda$. Path $\beta$ is never more than that distance away from one or the other of the legs of path $\alpha$. These distances are much smaller than the lengthscale of the potential, $R$. Therefore the scatterings of path $\beta$ are all correlated to scatterings on one or other leg of path $\alpha$. This means that ensemble averaging will not destroy the contribution of this pair of paths. To evaluate the contribution of pairs of paths of this type we construct a method based on that used by Argaman [5]. Since we are assuming that for every path $\alpha$, there is a path $\beta$, where $\alpha$ and $\beta$ are described above, we can assume the amplitude of path $\beta$ is approximately equal to that of path $\alpha$. However path $\beta$ has to go slightly further than path $\alpha$, because it crosses itself. This means we expect the action of path $\beta$ to be slightly greater than that of path $\alpha[5]$.

Note that the Hikami boxes in the standard diagrammatic approach are more complicated: they are 'dressed' by additional impurity lines, which is equivalent to having an extra quantum scattering inside the box. It is not clear why the contribution of such an extra scatterer should be important in the semiclassical approach, since its inclusion would be a small correction to the semiclassical Hikami box described above. However, the two methods are so different that such a direct comparison does not make much sense.

We now make the weak diagonal approximation, by assuming we can ignore all terms in the double summation in (4) which do not have the geometry described above. Clearly terms in the double sum with $\beta=\alpha$ have already been taken into account by the strong diagonal approximation. We can then substitute the following into (4),

$$
A_{\beta}=A_{\alpha} \quad S_{\beta}=S_{\alpha}+\delta S
$$

Since we are considering pairs of paths that are not identical, the double summation over $\alpha$ and $\beta$ in (4) gives two contributions for each pair of paths. For example if we label a given pair of paths, 1 and 2, then one gets a contribution to the double 
(a)

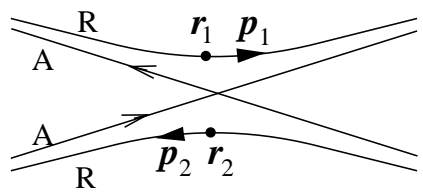

(b)

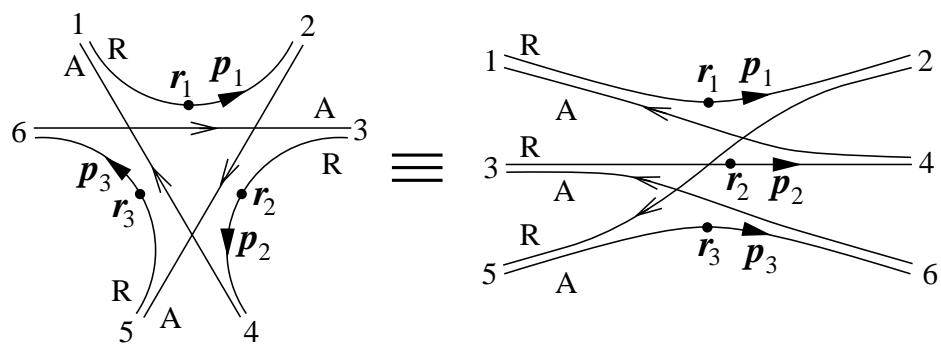

Figure 2. The Hikami boxes (the dashed boxes in Fig. 1) are shown here in more detail. In (a) $\boldsymbol{r}_{1}$ and $\boldsymbol{r}_{2}$ are close, while $\boldsymbol{p}_{1}$ and $\boldsymbol{p}_{2}$ are approximately anti-parallel. In (b) $\boldsymbol{r}_{1}, \boldsymbol{r}_{2}$ and $\boldsymbol{r}_{3}$ are close, while $\boldsymbol{p}_{1}, \boldsymbol{p}_{2}$ and $\boldsymbol{p}_{3}$ are approximately parallel. The directions of the arrows are explained in the caption to Fig. 1.

summation for $\alpha=1$ and $\beta=2$, and an identical contribution from $\alpha=2$ and $\beta=1$. The assumptions in (8) allow us to write the $i$ th term in the weak diagonal expansion of the spectral form factor as,

$$
K_{i}(t)=2 \sum_{\alpha_{i}}\left\langle\left|A_{\alpha_{i}}\right|^{2} \delta\left(t-T_{\alpha_{i}}\right) \exp \left[-i \delta S_{i} / \hbar\right]\right\rangle
$$

where the sum over $\alpha_{i}$ is over all periodic paths have $i$ loops. As can be seen in Fig. 1, to derive $K_{2}(t)$ and $K_{3}(t)$ we need the action difference in two geometries of Hikami box, these geometries are shown in Fig. 2. The first is one in which each path comes close to itself once (Fig. 2a) and the second is one in which each path comes close to a single point on itself twice (Fig. 2b). In the first case, each path enters the Hikami box twice, and the action difference between the two paths is,

$$
\delta S_{2}=\left(\boldsymbol{r}_{1}-\boldsymbol{r}_{2}\right) \cdot\left(\boldsymbol{p}_{2}+\boldsymbol{p}_{1}\right)
$$

In the second case, each path enters the Hikami box three times, the action difference is,

$$
\delta S_{3 a}=\left(\boldsymbol{r}_{1}-\boldsymbol{r}_{3}\right) \cdot \boldsymbol{p}_{1}+\left(\boldsymbol{r}_{2}-\boldsymbol{r}_{1}\right) \cdot \boldsymbol{p}_{2}+\left(\boldsymbol{r}_{3}-\boldsymbol{r}_{2}\right) \cdot \boldsymbol{p}_{3}
$$

The reason for the notation we have chosen for the subscripts on $\delta S_{2}$ and $\delta S_{3 a}$ will be made clear later in this paper.

\section{The two loop contribution in the weak diagonal expansion.}

Here we calculate two loop term in the weak diagonal expansion. This is the leading order correction to the strong diagonal approximation (1 loop) result. The geometry of paths that contribute to the two loop term is shown schematically in Fig. 1b. We expect this to be the dominant contribution to the weak diagonal perturbation expansion in a system with time-reversal symmetry. However in a system without time-reversal symmetry the two loop term in the weak diagonal expansion is zero. 
This is because one path follows the time-reverse of the other path for some of its period. In a system without time-reversal symmetry it is impossible for a path to exactly follow the time-reverse of another path.

In all the figures in this paper we have followed the Feynman diagram convention for the arrows. This means that the arrows on the retarded Green's functions (R) point in the direction of the underlying classical path, while the arrows on the advanced Green's functions (A) point in the opposite direction. This leads to the slightly counter intuitive observation that those pairs of paths which have the arrows pointing in opposite directions at all points are the only ones to survive in a system without time-reversal symmetry. In the language of disordered systems, the pairs of paths which have the arrows pointing in different directions are diffusons, while those with the arrows pointing in the same direction are Cooperons.

The two loop term in the weak diagonal expansion, shown schematically in Fig. 1b. can be found by substituting (10) into (9),

$$
K_{2}(t)=2 \sum_{\alpha}\left\langle\left|A_{\alpha}\right|^{2} \delta\left(t-T_{\alpha}\right) \exp \left[-\frac{i}{\hbar}\left(\boldsymbol{r}_{1}-\boldsymbol{r}_{2}\right) \cdot\left(\boldsymbol{p}_{2}+\boldsymbol{p}_{1}\right)\right]\right\rangle
$$

where the $\alpha$ th path is a path which starts at $(\boldsymbol{r}, \boldsymbol{p})$, passes through $\left(\boldsymbol{r}_{1}, \boldsymbol{p}_{1}\right)$ at time $t_{1}$, and $\left(\boldsymbol{r}_{2}, \boldsymbol{p}_{2}\right)$ at time $t_{2}$, before returning to $(\boldsymbol{r}, \boldsymbol{p})$ at time, $t$. The sum over $\alpha$ is over all primitive periodic paths and all repetitions of them, however in what follows we will ignore the possibility of repetitions. This is justified in [1] by noting that the number of primitive periodic paths grows exponentially with the period of the path. Therefore repetitions make a very small contribution to the sum over all paths with a given period.

The amplitude $A_{\alpha}$ is the same as in the derivation of $K_{1}(E, t)$, the following result was derived in [1],

$$
\left|A_{\alpha}\right|^{2} \delta\left(t-T_{\alpha}\right)=\frac{t}{(2 \pi \hbar)^{2}} \int \mathrm{d} \boldsymbol{r} \mathrm{d} \boldsymbol{p}_{E} f_{\alpha E}(\boldsymbol{r}, \boldsymbol{p}, t ; \boldsymbol{r}, \boldsymbol{p})
$$

where $f_{\alpha E}\left(\boldsymbol{r}^{\prime}, \boldsymbol{p}^{\prime}, t ; \boldsymbol{r}, \boldsymbol{p}\right)$ is the probability of a particle following path $\alpha$ from $(\boldsymbol{r}, \boldsymbol{p})$ to $\left(\boldsymbol{r}^{\prime}, \boldsymbol{p}^{\prime}\right)$ in a time $t$, given that the particle's final energy, $E$, is constrained to equal its initial energy. The integral over momentum is restricted to the energy surface, $\mathrm{d} \boldsymbol{p}_{E}$ is defined below (7). Equation (13) was originally derived for purely classical paths. We want to consider paths which are mainly classical, but which occasionally undergo a quantum scattering. For purely classical paths $f_{\alpha E}\left(\boldsymbol{r}^{\prime}, \boldsymbol{p}^{\prime}, t ; \boldsymbol{r}, \boldsymbol{p}\right)$ is simply a $\delta$-function at the point in phase space which a classical path starting at $(\boldsymbol{r}, \boldsymbol{p})$ would reach after time, $t$. We include the occasional quantum scattering event by dividing a given path up into segments of classical and quantum behaviour. The classical segments will provide most of the path, but there will be occasional short quantum segments. The classical segments can be related to the classical probability of propagating between two points in a given time. The quantum segments of the path have to be dealt with by finding the quantum mechanical propagator for that scattering event. If a quantum segment of the path goes from $\left(\boldsymbol{r}_{i}, \boldsymbol{p}_{i}\right)$ to $\left(\boldsymbol{r}_{i+1}, \boldsymbol{p}_{i+1}\right)$, this can be written in the form $A \exp [i R]$ where $A$ and $R$ are defined by the quantum mechanics of the scattering event, and are functions of $\left(\boldsymbol{r}_{i, i+1}, \boldsymbol{p}_{i, i+1}\right)$. An example of such a quantum scattering event is the penumbra diffraction of paths that graze semiclassically large scatterers, discussed in [28]. The probability of propagating from $\left(\boldsymbol{r}_{i}, \boldsymbol{p}_{i}\right)$ to $\left(\boldsymbol{r}_{i+1}, \boldsymbol{p}_{i+1}\right)$ is simply $|A|^{2}$. Since the scattering is not classical, this probability will not be a $\delta$-function. So a particle on a classical path encountering this quantum scatterer will be spread 
over a number of classical paths after leaving the quantum scattering region. The probabilities of propagating along each segment of the path can be multiplied together to give the probability of following the whole of that path. This procedure gives a result of the same form as (13), however the probability $f_{\alpha E}\left(\boldsymbol{r}^{\prime}, \boldsymbol{p}^{\prime}, t ; \boldsymbol{r}, \boldsymbol{p}\right)$ can now be written down for any path, whether it is purely classical or includes quantum scattering events, such as diffraction, tunnelling, etc. Similarly, when the sum over $\alpha$ is carried out it is now a sum over all the paths including quantum scattering events rather than just purely classical paths. In the limit of paths much longer than $t_{Q}$, a particle can be considered as mostly following classical paths, but with a small amount of diffusion from one classical path to those nearby in phase-space [6], where the timescale for this diffusion is set by $t_{Q}$.

Consider a quantity which is only non-zero in the vicinity of the $\alpha$ th isolated periodic path through the system. If one ignores repetitions of the path then the integration of that quantity over the surface in phase space with energy, $E$, can be written in terms of coordinates parallel, $\left(\boldsymbol{r}_{\|}, \boldsymbol{p}_{\|}\right)$, and perpendicular, $\left(\boldsymbol{r}_{\perp}, \boldsymbol{p}_{\perp}\right)$, to the path. Then,

$$
\begin{aligned}
& \int \mathrm{d} \boldsymbol{r}(\cdots)=\int \mathrm{d} r_{\|} \mathrm{d} \boldsymbol{r}_{\perp}(\cdots)=v_{E} \int_{0}^{t} \mathrm{~d} t^{\prime} \int \mathrm{d} \boldsymbol{r}_{\perp}(\cdots) \\
& \int \mathrm{d} \boldsymbol{p}_{E}(\cdots)=\int \mathrm{d} p_{\|} \mathrm{d} \boldsymbol{p}_{\perp} v_{E}^{-1} \delta\left[p_{\|}-p_{E}\right](\cdots)=v_{E}^{-1} \int \mathrm{d} \boldsymbol{p}_{\perp}(\cdots)
\end{aligned}
$$

where the $\mathrm{d} \boldsymbol{p}_{E}$ is defined just after equation (7). This was used in [1] to prove (6), and will be of great use to us.

The exponent in (12) is in terms of the two points $\left(\boldsymbol{r}_{1}, \boldsymbol{p}_{1}\right)$ and $\left(\boldsymbol{r}_{2}, \boldsymbol{p}_{2}\right)$, which are both somewhere on path $\alpha$. It is therefore necessary cast the right-hand side of (13) in a form which specifies that these points are indeed on path $\alpha$. We will now discuss the details of how this is done. Consider a path which starts at $(\boldsymbol{r}, \boldsymbol{p})$. We want to know the probability that the path passes through $\left(\boldsymbol{r}^{\prime}, \boldsymbol{p}^{\prime}\right)$. We do not care when the path passes through $\left(\boldsymbol{r}^{\prime}, \boldsymbol{p}^{\prime}\right)$, so long as it does so at some time between time $t_{a}$ and time $t_{b}$. So long as the period of the path is more than $t_{2}$, the probability density for this to occur is $\int_{t_{a}}^{t_{b}} \mathrm{~d} t^{\prime} f_{\alpha E}\left(\boldsymbol{r}^{\prime}, \boldsymbol{p}^{\prime}, t^{\prime} ; \boldsymbol{r}, \boldsymbol{p}\right)$. Therefore the probability of going from $\left(\boldsymbol{r}_{0}, \boldsymbol{p}_{0}\right)$ to $(\boldsymbol{r}, \boldsymbol{p})$ in time, t, and passing through $\left(\boldsymbol{r}^{\prime}, \boldsymbol{p}^{\prime}\right)$ on the way from $\left(\boldsymbol{r}_{0}, \boldsymbol{p}_{0}\right)$ to $(\boldsymbol{r}, \boldsymbol{p})$ is,

$$
\int_{0}^{t} \mathrm{~d} t^{\prime} f_{\alpha E}\left(\boldsymbol{r}, \boldsymbol{p}, t-t^{\prime} ; \boldsymbol{r}^{\prime}, \boldsymbol{p}^{\prime}\right) f_{\alpha E}\left(\boldsymbol{r}^{\prime}, \boldsymbol{p}^{\prime}, t^{\prime} ; \boldsymbol{r}_{0}, \boldsymbol{p}_{0}\right)
$$

Given that the propagation probability for the path $\alpha$ from $\left(\boldsymbol{r}_{0}, \boldsymbol{p}_{0}\right)$ to $(\boldsymbol{r}, \boldsymbol{p})$ in time $t$, is $f_{\alpha E}\left(\boldsymbol{r}, \boldsymbol{p}, t ; \boldsymbol{r}_{0}, \boldsymbol{p}_{0}\right)$, one can insist that the point $\left(\boldsymbol{r}^{\prime}, \boldsymbol{p}^{\prime}\right)$ is somewhere on the path. Then casting all coordinates in terms of components parallel and perpendicular to the path,

$$
\begin{aligned}
f_{\alpha E}\left(\boldsymbol{r}, \boldsymbol{p}, t ; \boldsymbol{r}_{0}, \boldsymbol{p}_{0}\right) & =\int \mathrm{d} \boldsymbol{r}_{\perp}^{\prime} \mathrm{d} \boldsymbol{p}_{\perp}^{\prime} \\
& \times \int_{0}^{t} \mathrm{~d} t^{\prime} f_{\alpha E}\left(\boldsymbol{r}, \boldsymbol{p}, t-t^{\prime} ; \boldsymbol{r}^{\prime}, \boldsymbol{p}^{\prime}\right) f_{\alpha E}\left(\boldsymbol{r}^{\prime}, \boldsymbol{p}^{\prime}, t^{\prime} ; \boldsymbol{r}_{0}, \boldsymbol{p}_{0}\right)
\end{aligned}
$$

The integral over all $\left(\boldsymbol{r}_{\perp}^{\prime}, \boldsymbol{p}_{\perp}^{\prime}\right)$ in the vicinity of the path $\alpha$ will pick up a $\delta$-function when $\left(\boldsymbol{r}^{\prime}, \boldsymbol{p}^{\prime}\right)$ is on the path, with no contribution the rest of the time. 
As previously mentioned we wish to specify that the points $\left(\boldsymbol{r}_{1}, \boldsymbol{p}_{1}\right)$ and $\left(\boldsymbol{r}_{2}, \boldsymbol{p}_{2}\right)$ are somewhere on path $\alpha$. Using (16) to specify this, (13) becomes,

$$
\begin{aligned}
\left|A_{\alpha}\right|^{2} \delta\left(t-T_{\alpha}\right)= & \frac{t}{(2 \pi \hbar)^{2}} \int_{\Gamma_{\alpha}} \mathrm{d} \boldsymbol{r} \mathrm{d} \boldsymbol{p}_{E} \int \mathrm{d} \boldsymbol{r}_{1 \perp} \mathrm{d} \boldsymbol{p}_{1 \perp} \mathrm{d} \boldsymbol{r}_{2 \perp} \mathrm{d} \boldsymbol{p}_{2 \perp} \\
& \times \int_{0}^{t} \mathrm{~d} t_{1} \int_{t_{1}}^{t+t_{1}} \mathrm{~d} t_{2} f_{\alpha E}\left(\boldsymbol{r}, \boldsymbol{p}, t ; \boldsymbol{r}_{2}, \boldsymbol{p}_{2}, t_{2} ; \boldsymbol{r}_{1}, \boldsymbol{p}_{1}, t_{1} ; \boldsymbol{r}, \boldsymbol{p}\right)
\end{aligned}
$$

where the integrals over $\boldsymbol{r}_{1,2}$ and $\boldsymbol{p}_{(1,2) E}$ are over the phase space surface with energy, $E$. We have defined $f_{\alpha E}\left(\boldsymbol{r}, \boldsymbol{p}, t ; \boldsymbol{r}_{2}, \boldsymbol{p}_{2}, t_{2} ; \boldsymbol{r}_{1}, \boldsymbol{p}_{1}, t_{1} ; \boldsymbol{r}, \boldsymbol{p}\right)$ as the probability that a classical particle with energy, $E$, which starts at $(\boldsymbol{r}, \boldsymbol{p})$, passes through $\left(\boldsymbol{r}_{1}, \boldsymbol{p}_{1}\right)$ at time, $t_{1}$, then through $\left(\boldsymbol{r}_{2}, \boldsymbol{p}_{2}\right)$ at time, $t_{2}$, before returning to $(\boldsymbol{r}, \boldsymbol{p})$ at time, $t$. Hence

$$
\begin{aligned}
& f_{\alpha E}\left(\boldsymbol{r}, \boldsymbol{p}, t ; \boldsymbol{r}_{2}, \boldsymbol{p}_{2}, t_{2} ; \boldsymbol{r}_{1}, \boldsymbol{p}_{1}, t_{1} ; \boldsymbol{r}, \boldsymbol{p}\right) \\
& \quad=f_{\alpha E}\left(\boldsymbol{r}, \boldsymbol{p}, t-t_{2} ; \boldsymbol{r}_{2}, \boldsymbol{p}_{2}\right) f_{\alpha E}\left(\boldsymbol{r}_{2}, \boldsymbol{p}_{2}, t_{2}-t_{1} ; \boldsymbol{r}_{1}, \boldsymbol{p}_{1}\right) f_{\alpha E}\left(\boldsymbol{r}_{1}, \boldsymbol{p}_{1}, t_{1} ; \boldsymbol{r}, \boldsymbol{p}\right)
\end{aligned}
$$

The integrals over $\boldsymbol{r}$ and $\boldsymbol{p}_{E}$ are carried out by noting that,

$\int \mathrm{d} \boldsymbol{r} \mathrm{d} \boldsymbol{p}_{E} f_{\alpha E}\left(\boldsymbol{r}, \boldsymbol{p}, t ; \boldsymbol{r}_{2}, \boldsymbol{p}_{2}, t_{2} ; \boldsymbol{r}_{1}, \boldsymbol{p}_{1}, t_{1} ; \boldsymbol{r}, \boldsymbol{p}\right)=f_{\alpha E}\left(\boldsymbol{r}_{1}, \boldsymbol{p}_{1}, t ; \boldsymbol{r}_{2}, \boldsymbol{p}_{2}, t^{\prime} ; \boldsymbol{r}_{1}, \boldsymbol{p}_{1}\right)$

where we have defined $t^{\prime} \equiv t_{2}-t_{1}$. The integrand is now independent of $t_{1}$, so the integral over it simply generates a factor of $t$. Substituting (19) and (17) into (12), we note that we only expect a contribution when $\boldsymbol{p}_{2}$ is approximately anti-parallel to $\boldsymbol{p}_{1}$. Therefore we can resolve $\boldsymbol{r}_{2}$ and $\boldsymbol{p}_{2}$ in the exponent of (12) into components parallel and perpendicular to the momentum $\boldsymbol{p}_{1}$. The integrals over $\boldsymbol{r}_{2 \perp}$ and $\boldsymbol{p}_{2 \perp}$ can then be evaluated using the following stationary phase approximation,

$$
\int \mathrm{d} x \mathrm{~d} p f(x, p) \exp [i x p / \hbar] \stackrel{\hbar \rightarrow 0}{\longrightarrow}(2 \pi \hbar) f(0,0)
$$

This means the the only contribution comes from paths where $\boldsymbol{r}_{2 \perp}=0$ and $\boldsymbol{p}_{2 \perp}=0$. The integral over $\boldsymbol{p}_{2 \|}$ is constrained by the energy $\delta$-function, so $\left|\boldsymbol{p}_{1}\right|=\left|\boldsymbol{p}_{2}\right|=p_{E}$, and therefore $\boldsymbol{p}_{2}=-\boldsymbol{p}_{1}$. Hence we find,

$$
\begin{aligned}
\left|A_{\alpha}\right|^{2} \delta\left(t-T_{\alpha}\right) & \exp \left[-i \delta S_{2} / \hbar\right] \\
= & \frac{t^{2}}{(2 \pi \hbar)^{3-d}} \int \mathrm{d} \boldsymbol{r}_{1 \perp} \mathrm{d} \boldsymbol{p}_{1 \perp} \int_{0}^{t} \mathrm{~d} t^{\prime} f_{\alpha E}\left(\boldsymbol{r}_{1}, \boldsymbol{p}_{1}, t ; \boldsymbol{r}_{1},-\boldsymbol{p}_{1}, t^{\prime} ; \boldsymbol{r}_{1}, \boldsymbol{p}_{1}\right)
\end{aligned}
$$

where $\delta S_{2}$ is given by (10). The integral over $\boldsymbol{r}_{1 \perp}$ and $\boldsymbol{p}_{1 \perp}$ can be converted back to the system's coordinates by noting that since the integral over $t^{\prime}$ makes the right-hand side of (21) independent of $r_{\|}^{\prime}$, the result is unchanged if we integrate over $r_{\|}^{\prime}$, so long as we divide by the path length, $v_{E} t$. Then (14) can be used to put the integrals in terms of the systems' coordinates. However when we do this, the integral of $\boldsymbol{r}_{1}$ and $\boldsymbol{p}_{1 E}$ over all phase space double-counts all the paths, so we have to divide through by two. Since we get a double contribution from each path if the system has time-reversal symmetry and no contribution if it does not, we multiply through by $2(2-\beta)$. Hence,

$$
\begin{aligned}
\left|A_{\alpha}\right|^{2} \delta\left(t-T_{\alpha}\right) & \exp \left[-i \delta S_{2} / \hbar\right] \\
& =\frac{(2-\beta) t}{(2 \pi \hbar)^{3-d}} \int \mathrm{d} \boldsymbol{r}_{1} \mathrm{~d} \boldsymbol{p}_{1 E} \int_{0}^{t} \mathrm{~d} t^{\prime} f_{\alpha E}\left(\boldsymbol{r}_{1}, \boldsymbol{p}_{1}, t ; \boldsymbol{r}_{1},-\boldsymbol{p}_{1}, t^{\prime} ; \boldsymbol{r}_{1}, \boldsymbol{p}_{1}\right)
\end{aligned}
$$

Finally substituting this into (12), summing over all possible paths, and carrying out the averaging $\langle\cdots\rangle$, we find,

$$
K_{2}(E, t)=\frac{2(2-\beta) t}{(2 \pi \hbar)^{3-d}} \int \mathrm{d} \boldsymbol{r}_{1} \mathrm{~d} \boldsymbol{p}_{1 E} \int_{0}^{t} \mathrm{~d} t^{\prime}\left\langle f_{E}\left(\boldsymbol{r}_{1}, \boldsymbol{p}_{1}, t ; \boldsymbol{r}_{1},-\boldsymbol{p}_{1}, t^{\prime} ; \boldsymbol{r}_{1}, \boldsymbol{p}_{1}\right)\right\rangle
$$


At present we have not specified what form the averaging $\langle\cdots\rangle$ will take in the definition of the SFF and the TLCF. However it is clear from (23) that it only affects the propagation probability. So if the averaging were over an energy window near the energy, $E$, then one only needs to know what the propagation probability is after it has been averaged it over that energy window. In this paper we will be applying the above result to a disordered system, so we can chose to define the averaging in the definition of the TLCF (1), and hence the SFF (4), as averaging over an ensemble of systems with different positions of the scatterers. In this case the averaging of the propagation probability in (23) would be over this ensemble of systems.

Equation (23) is the two loop term in the weak diagonal expansion of the SFF. When we set $\beta=1$, it applies to a system with time-reversal symmetry, and is the leading order correction to the strong diagonal approximation. When we set $\beta=2$, it applies to a system without time-reversal symmetry, for which the two loop term is zero. Therefore in the $\beta=2$ case, it is the three loop term which is the leading order correction to the strong diagonal approximation.

\section{The three loop contribution to the weak diagonal expansion (for a system without time-reversal symmetry).}

In this paper we are interested in calculating the leading order correction to the strong diagonal approximation. As discussed at the beginning of the previous section, the two loop term of the weak diagonal perturbation expansion is zero in a system without time-reversal symmetry. Therefore, to find the leading correction to the strong diagonal approximation for a system without time-reversal symmetry, it is necessary to calculate the three loop term in the expansion. In general there are five path geometries that contribute to the three loop term in the expansion, however we are only interested in the three loop term for a system without time-reversal symmetry. In such a system three of these five possible geometries give no contribution because one path follows the time reverse of the other at some point in their period. This means we have the contribution of two geometries to calculate, these geometries are shown in Fig. 1c,d.

The first contribution is shown in Fig. 1c, the action difference between the two paths, $\delta S_{3 a}$, is given by (11). The amplitude, $A_{\alpha}$, is given by taking (13) and using (16) to insist that $\left(\boldsymbol{r}_{1}, \boldsymbol{p}_{1}\right),\left(\boldsymbol{r}_{2}, \boldsymbol{p}_{2}\right)$ and $\left(\boldsymbol{r}_{3}, \boldsymbol{p}_{3}\right)$ are somewhere on the path $\alpha$,

$$
\begin{aligned}
&\left|A_{\alpha}\right|^{2} \delta\left(t-T_{\alpha}\right)=\frac{t}{(2 \pi \hbar)^{2}} \int_{\Gamma_{\alpha}} \mathrm{d} \boldsymbol{r} \mathrm{d} \boldsymbol{p}_{E} \int \mathrm{d} \boldsymbol{r}_{1 \perp} \mathrm{d} \boldsymbol{p}_{1 \perp} \mathrm{d} \boldsymbol{r}_{2 \perp} \mathrm{d} \boldsymbol{p}_{2 \perp} \mathrm{d} \boldsymbol{r}_{3 \perp} \mathrm{d} \boldsymbol{p}_{3 \perp} \\
& \quad \times \int_{0}^{t} \mathrm{~d} t_{1} \int_{t_{1}}^{t+t_{1}} \mathrm{~d} t_{2} \int_{t_{2}}^{t+t_{1}} \mathrm{~d} t_{3} f_{\alpha E}\left(\boldsymbol{r}, \boldsymbol{p}, t ; \boldsymbol{r}_{3}, \boldsymbol{p}_{3}, t_{3} ; \boldsymbol{r}_{2}, \boldsymbol{p}_{2}, t_{2} ; \boldsymbol{r}_{1}, \boldsymbol{p}_{1}, t_{1} ; \boldsymbol{r}, \boldsymbol{p}\right)
\end{aligned}
$$

The integral over $(\boldsymbol{r}, \boldsymbol{p})$ is carried out using (19). We only expect contributions when $\boldsymbol{p}_{2}$ and $\boldsymbol{p}_{3}$ are approximately parallel to $\boldsymbol{p}_{1}$. So substituting (11) and (24) into (9), we carry out the stationary phase integrals over $\left(\boldsymbol{r}_{2 \perp}, \boldsymbol{p}_{2 \perp}\right)$ and $\left(\boldsymbol{r}_{3 \perp}, \boldsymbol{p}_{3 \perp}\right)$, this leaves the integral over $\left(\boldsymbol{r}_{1 \perp}, \boldsymbol{p}_{1 \perp}\right)$. As in the two loop case we introduce an extra integral over $r_{1 \|}$ and divide through by the length of the path, $v_{E} t$, the integral of $\boldsymbol{r}_{1}$ and $\boldsymbol{p}_{1 E}$ is then over the phase space surface with energy, $E$. However now this integral over all phase space counts each contributing path three times, so we must divide through by three. For completeness we also multiply the result by the time-reversal symmetry factor, $2 / \beta$, so that when $\beta=2(\beta=1)$ we count each path once (twice). However one should note that to calculate the total contribution in a system with time-reversal 
symmetry, one would not only have to set $\beta=1$ in these equations. One also has to calculate the contribution of the trajectories that we ignore because they are zero in systems without time-reversal symmetry. Summing over all paths and carrying out the averaging $\langle\cdots\rangle$, we find,

$$
\begin{aligned}
K_{3 a}(E, t)=\frac{4 t}{3 \beta(2 \pi \hbar)^{4-2 d}} \int \mathrm{d} \boldsymbol{r}_{1} \mathrm{~d} \boldsymbol{p}_{1 E} \int_{0}^{t} \mathrm{~d} t_{1}^{\prime} \int_{0}^{t-t_{1}^{\prime}} \mathrm{d} t_{2}^{\prime} \\
\quad \times\left\langle f_{E}\left(\boldsymbol{r}_{1}, \boldsymbol{p}_{1}, t ; \boldsymbol{r}_{1}, \boldsymbol{p}_{1}, t_{2}^{\prime} ; \boldsymbol{r}_{1}, \boldsymbol{p}_{1}, t_{1}^{\prime} ; \boldsymbol{r}_{1}, \boldsymbol{p}_{1}\right)\right\rangle
\end{aligned}
$$

where we have defined $t_{1}^{\prime} \equiv t_{2}-t_{1}$ and $t_{2}^{\prime} \equiv t_{3}-t_{2}$.

The second contribution to the three loop term is shown in Fig. 1d, the action difference between the two paths is,

$$
\delta S_{3 b}=\left(\boldsymbol{r}_{1}-\boldsymbol{r}_{3}\right) \cdot\left(\boldsymbol{p}_{3}-\boldsymbol{p}_{1}\right)-\left(\boldsymbol{r}_{2}-\boldsymbol{r}_{4}\right) \cdot\left(\boldsymbol{p}_{4}-\boldsymbol{p}_{2}\right)
$$

and the amplitude can be written in terms of probabilities, by taking (13) and using (16) to insist that $\left(\boldsymbol{r}_{1}, \boldsymbol{p}_{1}\right),\left(\boldsymbol{r}_{2}, \boldsymbol{p}_{2}\right),\left(\boldsymbol{r}_{3}, \boldsymbol{p}_{3}\right)$ and $\left(\boldsymbol{r}_{4}, \boldsymbol{p}_{4}\right)$ are somewhere on the path $\alpha$,

$$
\begin{aligned}
\left|A_{\alpha}\right|^{2} \delta\left(t-T_{\alpha}\right)= & \frac{t}{(2 \pi \hbar)^{2}} \int_{\Gamma_{\alpha}} \mathrm{d} \boldsymbol{r} \mathrm{d} \boldsymbol{p}_{E} \int \mathrm{d} \boldsymbol{r}_{1 \perp} \mathrm{d} \boldsymbol{p}_{1 \perp} \mathrm{d} \boldsymbol{r}_{2 \perp} \mathrm{d} \boldsymbol{p}_{2 \perp} \mathrm{d} \boldsymbol{r}_{3 \perp} \mathrm{d} \boldsymbol{p}_{3 \perp} \mathrm{d} \boldsymbol{r}_{4 \perp} \mathrm{d} \boldsymbol{p}_{4 \perp} \\
& \times \int_{0}^{t} \mathrm{~d} t_{1} \int_{t_{1}}^{t+t_{1}} \mathrm{~d} t_{2} \int_{t_{2}}^{t+t_{1}} \mathrm{~d} t_{3} \int_{t_{3}}^{t+t_{1}} \mathrm{~d} t_{4} \\
& \times f_{\alpha E}\left(\boldsymbol{r}, \boldsymbol{p}, t ; \boldsymbol{r}_{4}, \boldsymbol{p}_{4}, t_{4} ; \boldsymbol{r}_{3}, \boldsymbol{p}_{3}, t_{3} ; \boldsymbol{r}_{2}, \boldsymbol{p}_{2}, t_{2} ; \boldsymbol{r}_{1}, \boldsymbol{p}_{1}, t_{1} ; \boldsymbol{r}, \boldsymbol{p}\right)
\end{aligned}
$$

The integral over $(\boldsymbol{r}, \boldsymbol{p})$ is carried out using (19). We only expect contributions when $\boldsymbol{p}_{3}$ is approximately parallel to $\boldsymbol{p}_{1}$, while $\boldsymbol{p}_{4}$ is approximately parallel to $\boldsymbol{p}_{2}$. Substituting (26) and (27) into (9), we carry out the stationary phase integrals over $\left(\boldsymbol{r}_{3 \perp}, \boldsymbol{p}_{3 \perp}\right)$ and $\left(\boldsymbol{r}_{4}, \boldsymbol{p}_{4}\right)$. This leaves the integral over $\left(\boldsymbol{r}_{1 \perp}, \boldsymbol{p}_{1 \perp}\right)$ and $\left(\boldsymbol{r}_{2 \perp}, \boldsymbol{p}_{2 \perp}\right)$, we turn these into integrals over the phase space surface with energy, $E$, by introducing extra integrals over $r_{1 \|}$ and $r_{2 \|}$, while dividing through twice by the path length. However now the integrals of $\boldsymbol{r}_{1,2}$ and $\boldsymbol{p}_{(1,2) E}$ over all phase space count each contributing path four times, so we must divide through by four. As before, we also multiply the result by the time-reversal symmetry factor, $2 / \beta$. Summing over all paths and carrying out the averaging $\langle\cdots\rangle$,

$$
\begin{gathered}
K_{3 b}(E, t)=\frac{1}{\beta(2 \pi \hbar)^{4-2 d}} \int \mathrm{d} \boldsymbol{r}_{1} \mathrm{~d} \boldsymbol{p}_{1 E} \mathrm{~d} \boldsymbol{r}_{2} \mathrm{~d} \boldsymbol{p}_{2 E} \int_{0}^{t} \mathrm{~d} t_{1}^{\prime} \int_{0}^{t-t_{1}^{\prime}} \mathrm{d} t_{2}^{\prime} \int_{0}^{t-t_{1}^{\prime}-t_{2}^{\prime}} \mathrm{d} t_{3}^{\prime} \\
\times\left\langle f_{E}\left(\boldsymbol{r}_{1}, \boldsymbol{p}_{1}, t ; \boldsymbol{r}_{2}, \boldsymbol{p}_{2}, t_{3}^{\prime} ; \boldsymbol{r}_{1}, \boldsymbol{p}_{1}, t_{2}^{\prime} ; \boldsymbol{r}_{2}, \boldsymbol{p}_{2}, t_{1}^{\prime} ; \boldsymbol{r}_{1}, \boldsymbol{p}_{1}\right)\right\rangle
\end{gathered}
$$

where we have defined $t_{1}^{\prime} \equiv t_{2}-t_{1}, t_{2}^{\prime} \equiv t_{3}-t_{2}$ and $t_{3}^{\prime} \equiv t_{4}-t_{3}$. The sum of (25) and (28) gives the three loop term in the weak diagonal perturbation expansion of the SFF for a system without time reversal symmetry. Since for such a system the two loop term in the expansion is zero, this is the leading order correction to the strong diagonal approximation.

\section{The leading order behaviour of the TLCF in a two-dimensional disordered system with time-reversal symmetry.}

We will now apply the results of the weak diagonal expansion to a two-dimensional dilute system of randomly placed semiclassical scatterers. Each scatterer is 
semiclassical in the sense that its radius, $a$, is much greater than the wavelength, $\lambda_{F}$. The typical distance between the scatterers is much larger than their radius.

In any given system the scatterers are randomly placed. We will be ensemble averaging our results, where the ensemble to be averaged over is an ensemble of systems with the same macroscopic properties but with different positions of the scatterers. The propagation probabilities through two separate regions of the potential are uncorrelated because the scatterers are randomly placed, and can therefore be ensemble averaged separately. We will assume,

$$
\begin{aligned}
\left\langlef _ { E } \left(\boldsymbol{r}_{1}, \boldsymbol{p}_{1}, t ; \boldsymbol{r}_{1},\right.\right. & \left.\left.-\boldsymbol{p}_{1}, t^{\prime} ; \boldsymbol{r}_{1}, \boldsymbol{p}_{1}\right)\right\rangle \\
& =\left\langle f_{E}\left(\boldsymbol{r}_{1}, \boldsymbol{p}_{1}, t-t^{\prime} ; \boldsymbol{r}_{1},-\boldsymbol{p}_{1}\right)\right\rangle\left\langle f_{E}\left(\boldsymbol{r}_{1},-\boldsymbol{p}_{1}, t^{\prime} ; \boldsymbol{r}_{1}, \boldsymbol{p}_{1}\right)\right\rangle
\end{aligned}
$$

where $\langle\cdots\rangle$ now denotes ensemble averaging. This assumption will be valid so long as $t, t^{\prime} \gg t_{\text {Erh }}$, because then the region over which the parts of the paths are correlated is a small proportion of the whole path.

On these relatively long timescales, the classical behaviour of the system is diffusive. The propagation probability, $f_{E}\left(\boldsymbol{r}^{\prime}, \boldsymbol{p}^{\prime}, t ; \boldsymbol{r}, \boldsymbol{p}\right)$, is not entirely classical, it include some diffractive scatterings, however these are not going to change the diffusive nature of the propagation probability distribution. After a small number of scatterings the direction of the momentum of a classical path is effectively randomised. Therefore the ensemble averaged propagation probability is independent of both the initial and final direction of the momentum. Hence,

$$
\left\langle f_{E}\left(\boldsymbol{r}^{\prime}, \boldsymbol{p}^{\prime}, t ; \boldsymbol{r}, \boldsymbol{p}\right)\right\rangle=W\left(\boldsymbol{r}^{\prime}, \boldsymbol{r} ; t\right) \frac{v_{E}}{S_{d} p_{E}^{d-1}}
$$

where $S_{d}$ is the surface area of a $d$-dimensional unit sphere. $W\left(\boldsymbol{r}, \boldsymbol{r}^{\prime} ; t\right)$ is the probability of a diffusing particle which starts at $\boldsymbol{r}$ being within $\mathrm{d} \boldsymbol{r}^{\prime}$ of $\boldsymbol{r}^{\prime}$ after time, $t$, it therefore satisfies the diffusion equation,

$$
\left[\frac{\partial}{\partial t}-D \nabla_{\boldsymbol{r}^{\prime}}^{2}\right] W\left(\boldsymbol{r}^{\prime}, \boldsymbol{r} ; t\right)=\delta(t) \delta\left(\boldsymbol{r}^{\prime}-\boldsymbol{r}\right)
$$

For time scales smaller than the ergodic time, $t_{\mathrm{erg}}=L^{2} / D$, the system is in the diffusive regime, the diffusing probability distribution $W\left(\boldsymbol{r}^{\prime}, \boldsymbol{r} ; t\right)$ does not know about the boundaries of the container and,

$$
W\left(\boldsymbol{r}^{\prime}, \boldsymbol{r} ; t\right)=W\left(\boldsymbol{r}^{\prime}-\boldsymbol{r} ; t\right)=(4 \pi D t)^{-d / 2} \exp \left[-\left|\boldsymbol{r}^{\prime}-\boldsymbol{r}\right|^{2} /(4 D t)\right]
$$

Disordered systems are believed to obey one-parameter scaling, hence it is usual to characterise disordered systems in terms of a single parameter, their dimensionless conductance, $g$. This is simply the conductance measured in units of $e^{2} /\left(n_{d} \hbar\right)$, where the parameter $n_{d}=\pi(2 \pi)^{d} / S_{d}$. The dimensionless conductance of a disordered system with volume, $L^{d}$, and diffusion coefficient, $D$, is given by,

$$
g=n_{d} E_{T h} / \Delta
$$

where the Thouless energy, $E_{T h}=\hbar / t_{\text {erg. }}$. We will therefore write our results for disordered systems in terms of the dimensionless conductance.

If one uses this description of a diffusive system to calculate the strong diagonal approximation of the SFF (6), one find that in two dimensions the result is $t$ independent. Since the TLCF is the Fourier transform of the SFF, the strong diagonal approximation of the TLCF is zero. This means that it is the corrections to the strong diagonal approximation that give the behaviour of the TLCF in a two dimensional diffusive systems. Here we will apply the weak diagonal approximation results derived 
in the previous sections to a diffusive system with time-reversal symmetry. By using the above description of propagation in a diffusive system, we calculate the two loop term in the weak diagonal perturbation expansion for such a system. This will give the leading order behaviour of the TLCF in a two dimensional diffusive system with time-reversal symmetry.

We substitute (30) and (23) into (5), and find the semiclassical evaluation of the two loop contribution to the TLCF, $R_{2}^{\mathrm{sc}}(\omega)$, is given by,

$$
\begin{aligned}
& R_{2}^{\mathrm{sc}}(\omega)=8(2-\beta) L^{2 d}\left(\frac{\Delta}{2 \pi \hbar}\right)^{3} \\
& \Re \mathrm{e}\left[\int_{0}^{\infty} \mathrm{d} t t \exp [i \omega t / \hbar] \int_{0}^{t} \mathrm{~d} t^{\prime} W\left(0 ; t^{\prime}\right) W\left(0 ; t-t^{\prime}\right)\right]
\end{aligned}
$$

where the level spacing, $\Delta$, is simply given by $\Delta=h^{d} v_{E} /\left(S_{d} p_{E}^{d-1} L^{d}\right)$, where $S_{d} p_{E}^{d-1} L^{d} / v_{E}$ is the volume of the constant energy surface of the phase space of the system.

Thus substituting (32) into (34), gives $R_{2}^{\mathrm{sc}}(\omega)$ in the diffusive regime,

$$
\begin{aligned}
& R_{2}^{\mathrm{sc}}(\omega)=\frac{8(2-\beta) L^{2 d}}{(4 \pi D)^{d}}\left(\frac{\Delta}{2 \pi \hbar}\right)^{3} \\
& \Re \mathrm{e}\left[\int_{0}^{\infty} \mathrm{d} t t \exp [i \omega t / \hbar] \int_{0}^{t} \mathrm{~d} t^{\prime} t^{\prime-d / 2}\left(t-t^{\prime}\right)^{-d / 2}\right]
\end{aligned}
$$

One can evaluate the integral over $t^{\prime}$ by noting it is a Euler $\beta$-function and so can be written in terms of $\Gamma$-functions. The result in 2 dimensions can be found by setting $d=2+\epsilon$, and expanding the $\Gamma$-functions in $\epsilon$.

However to compare the results of the semiclassics with those of the impurity diagram technique, we can Fourier transform the "W"s in (34) and then compare directly the functional forms of the results in the two approaches. If we define $W(\boldsymbol{q}, \omega)$ using,

$$
W(\boldsymbol{r} ; t)=\int \frac{\mathrm{d}^{d} \boldsymbol{q} \mathrm{d} \omega}{(2 \pi \hbar)^{d+1}} \exp [-i(\boldsymbol{q} \cdot \boldsymbol{r}+\omega t) / \hbar] W(\boldsymbol{q}, \omega)
$$

then in the diffusive regime,

$$
W(\boldsymbol{q}, \omega)=\hbar^{2}\left[D \boldsymbol{q}^{2}-i \hbar \omega\right]^{-1}
$$

Substituting (36) and (37) into (34), and carrying out the integrals over $t$ and $t^{\prime}$, we find the semiclassical result for the two loop term in the weak diagonal perturbation expansion,

$$
R_{2}^{\mathrm{sc}}(\omega)=8(2-\beta) \hbar L^{2 d}\left(\frac{\Delta}{2 \pi \hbar}\right)^{3} \Re \mathrm{e}\left[\frac{\partial\left[I_{1}(\omega)\right]^{2}}{\partial(i \omega)}\right]
$$

in deriving this we have defined,

$$
\begin{aligned}
I_{n}(\omega) & \equiv \int \frac{\mathrm{d}^{d} \boldsymbol{q}}{(2 \pi \hbar)^{d}} \hbar^{2 n}\left[D \boldsymbol{q}^{2}-i \hbar \omega\right]^{-n} \\
& =\frac{\Gamma(n-d / 2)}{(4 \pi)^{d / 2}(n-1) !}\left(\frac{\hbar}{\Delta}\right)^{n}\left(\frac{n_{d}}{L^{2} g}\right)^{d / 2}\left(-i \frac{\omega}{\Delta}\right)^{d / 2-n}
\end{aligned}
$$

where $g$ is the dimensionless conductance given by (33), and $\Delta$ is the average level spacing. 


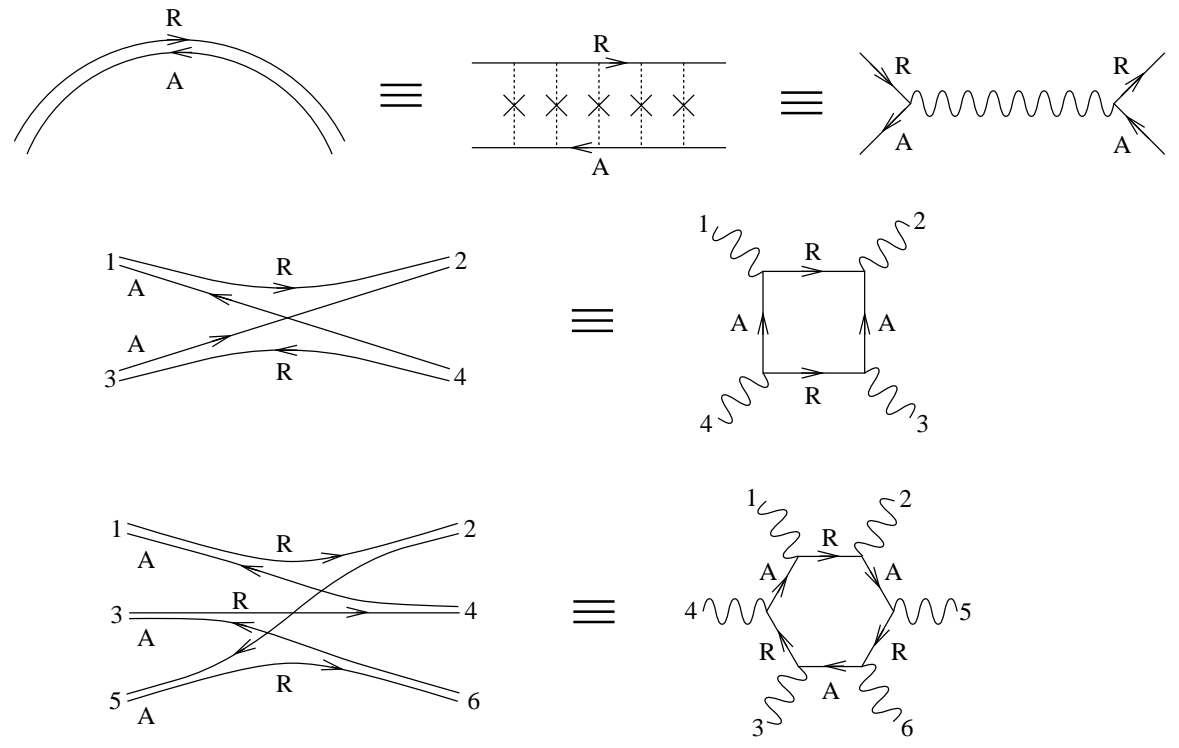

Figure 3. This dictionary allowing a translation from the trajectory pictures to the diagrams in the impurity diagram technique. A region where the two paths are identical corresponds to a diffuson (or a Cooperon, if one of the arrows is reversed). The semiclassical Hikami boxes are shown with the corresponding Hikami boxes in the impurity diagram technique.

We will now calculate the equivalent term in the impurity diagram technique $[29,17,24]$. The trajectory pictures used in the semiclassical approach (see Fig. 1 and 2) can easily be mapped onto the diagrams in the impurity diagram technique [23]. A dictionary for converting between the two is shown in Fig. 3. However this correspondence is fairly superficial, the picture drawn in the two approaches maybe similar, but the approach to calculations is different. Two dimensions is the critical dimensionality for the impurity diagram technique [16]. As a result renormalisation ideas apply, and one can ignore short trajectories and short range behaviour (in other words the large $\omega$ and $\boldsymbol{q}$ behaviour). By ignoring these ultraviolet divergences, the impurity diagram technique calculations are greatly simplified.

The two loop term in the impurity diagram technique is given by the trajectories shown in Fig. 1b, which can be converted to the more usual diagrammatics picture using Fig. 3. After throwing away the ultraviolet divergences, the two loop contribution is,

$$
R_{2}(\omega)=4(2-\beta) \hbar L^{2 d}\left(\frac{\Delta}{2 \pi \hbar}\right)^{3} \Re \mathrm{e}\left[\frac{\partial^{2}}{\partial(i \omega)^{2}}\left[i \omega\left(I_{1}(\omega)\right)^{2}\right]\right]
$$

carrying out one of the differentials over $\omega$,

$R_{2}(\omega)=4(2-\beta) \hbar L^{2 d}\left(\frac{\Delta}{2 \pi \hbar}\right)^{3} \Re \mathrm{e}\left[\frac{\partial}{\partial(i \omega)}\left[\left(I_{1}(\omega)\right)^{2}+\frac{2 i \omega}{\hbar} I_{1}(\omega) I_{2}(\omega)\right]\right]$

Since the divergent terms can only be ignored in the vicinity of the critical dimension, $d=2$, one must remember that (40) and hence (41), are only true for $d=2+\epsilon$ where $\epsilon$ is small. 
The functional form of the results of the two approaches, (38) and (41), are different. The impurity diagram technique result has an extra term, however it so happens that in two dimensions, this term is purely imaginary and so does not contribute to the TLCF. This is most easily shown by evaluating its contribution in $d=2+\epsilon$, writing the integrals in terms of $\Gamma$-functions and then taking $\epsilon$ to zero. Therefore in two dimensions the two approaches have the same functional form. In two dimensions the semiclassical result simply disagrees with the impurity diagram result by a factor of two. Since the impurity diagram technique result (41) is only true for two dimensions, our conclusion is that the semiclassics gets the right answer but with the prefactor wrong by a factor of two. However it looks suspiciously like it is so close to the correct result only as the result of a lucky cancellation of the term in the impurity diagram technique that the semiclassics can not reproduce. To confirm if this is the case we need to go to the next order in the weak diagonal expansion, and compare that result to the three loop term in the impurity diagram technique.

For the sake of completeness we now evaluate $R_{2}^{\mathrm{sc}}(\omega)$ in $d=2$, taking (38) setting $d=2+\epsilon$ where $\epsilon$ is small,

$$
R_{2}^{\mathrm{sc}}(s \Delta)=-\frac{(2-\beta)}{\pi g^{2+\epsilon}} \frac{1}{\epsilon} \Re \mathrm{e}\left[(-i s)^{\epsilon-1}\right] \stackrel{\epsilon \rightarrow 0}{\longrightarrow}-\frac{(2-\beta)}{2 g^{2}|s|}
$$

where $I_{1}(\omega)$ was evaluated using $(39), g$ is the dimensionless conductance given by (33), $s=\omega / \Delta, \beta=1(\beta=2)$ for a system with (without) time-reversal symmetry. As we have stated, this result is a factor of two larger than the equivalent impurity diagram result.

\section{The leading order behaviour of the TLCF in a two-dimensional disordered system without time-reversal symmetry.}

We now evaluate the first of the two semiclassical contributions to the three loop term in the weak diagonal expansion of the TLCF, $R_{3 a}^{\mathrm{sc}}(\omega)$, for a disordered system without time-reversal symmetry in the diffusive regime. Substituting (29) and (30) into (25), we find,

$$
\begin{aligned}
R_{3 a}^{\mathrm{sc}}(\omega)=\frac{16 L^{3 d}}{3 \beta} & \left(\frac{\Delta}{2 \pi \hbar}\right)^{4} \Re\left[\int_{0}^{\infty} \mathrm{d} t t \exp [i \omega t / \hbar]\right. \\
& \left.\times \int_{0}^{t} \mathrm{~d} t_{1}^{\prime} \mathrm{d} t_{2}^{\prime} W\left(0 ; t_{1}^{\prime}\right) W\left(0 ; t_{2}^{\prime}\right) W\left(0 ; t-t_{1}^{\prime}-t_{2}^{\prime}\right)\right]
\end{aligned}
$$

In the diffusive regime, one can use (32) to show this becomes,

$$
\begin{gathered}
R_{3 a}^{\mathrm{sc}}(\omega)=\frac{16 L^{3 d}}{3 \beta(4 \pi D)^{3 d / 2}}\left(\frac{\Delta}{2 \pi \hbar}\right)^{4} \Re \mathrm{e}\left[\int_{0}^{\infty} \mathrm{d} t t \exp [i \omega t / \hbar]\right. \\
\left.\times \int_{0}^{t} \mathrm{~d} t_{1}^{\prime} \mathrm{d} t_{2}^{\prime}\left[t_{1}^{\prime} t_{2}^{\prime}\left(t-t_{2}^{\prime}-t_{1}^{\prime}\right)\right]^{-d / 2}\right]
\end{gathered}
$$

However as before we Fourier transform (43) to the $(\boldsymbol{q}, \omega)$-representation to allow comparison with the impurity diagram technique. Substituting (36) and (37) into (43) and carrying out the integrals over $t$ and $t_{1,2}^{\prime}$,

$$
R_{3 a}^{\mathrm{sc}}(\omega)=\frac{16 \hbar L^{3 d}}{3 \beta}\left(\frac{\Delta}{2 \pi \hbar}\right)^{4} \Re \mathrm{e}\left[\frac{\partial}{\partial(i \omega)}\left[I_{1}(\omega)\right]^{3}\right]
$$


In $(2+\epsilon)$ dimensions where $\epsilon$ is small, this becomes,

$$
R_{3 a}^{\mathrm{sc}}(s \Delta)=\frac{1}{6 \pi \beta g^{3}} \frac{1}{\epsilon^{2}} \Re \mathrm{e}\left[(-i s)^{(3 \epsilon / 2)-1}\right]
$$

We now evaluate the second of the two semiclassical contributions, $R_{3 b}^{\mathrm{sc}}(\omega)$, for a disordered system without time-reversal symmetry in the diffusive regime. Substituting (29) and (30) into (28), we find,

$$
\begin{aligned}
R_{3 b}^{\mathrm{sc}}(\omega)=\frac{4 L^{3 d}}{\beta} & \left(\frac{\Delta}{2 \pi \hbar}\right)^{4} \Re \mathrm{e}\left[\int \mathrm{d} \boldsymbol{r}\right. \\
& \times \int_{0}^{\infty} \mathrm{d} t t \exp [i \omega t / \hbar] \int_{0}^{t} \frac{\mathrm{d} t_{1}^{\prime}}{t} \int_{0}^{t-t_{1}^{\prime}} \mathrm{d} t_{2}^{\prime} \int_{0}^{t-t_{1}^{\prime}-t_{2}^{\prime}} \mathrm{d} t_{3}^{\prime} \\
& \left.\times W\left(\boldsymbol{r} ; t_{1}^{\prime}\right) W\left(-\boldsymbol{r} ; t_{2}^{\prime}\right) W\left(\boldsymbol{r} ; t_{3}^{\prime}\right) W\left(-\boldsymbol{r} ; t-t_{1}^{\prime}-t_{2}^{\prime}-t_{3}^{\prime}\right)\right]
\end{aligned}
$$

where $\boldsymbol{r}=\boldsymbol{r}_{2}-\boldsymbol{r}_{1}$. Substituting in (32) this becomes,

$$
\begin{aligned}
R_{3 b}^{\mathrm{sc}}(\omega)=\frac{2 L^{3 d}}{\beta(4 \pi D)^{2 d}} & \left(\frac{\Delta}{2 \pi \hbar}\right)^{4} \Re \mathrm{e}\left[\int \mathrm{d} \boldsymbol{r} \int_{0}^{\infty} \mathrm{d} t t \exp [i \omega t / \hbar]\right. \\
& \times \int_{0}^{t} \frac{\mathrm{d} t_{1}^{\prime}}{t} \int_{0}^{t-t_{1}^{\prime}} \mathrm{d} t_{2}^{\prime} \int_{0}^{t-t_{1}^{\prime}-t_{2}^{\prime}} \mathrm{d} t_{3}^{\prime}\left[t_{1}^{\prime} t_{2}^{\prime} t_{3}^{\prime}\left(t-t_{3}^{\prime}-t_{2}^{\prime}-t_{1}^{\prime}\right)\right]^{-d / 2} \\
& \left.\times \exp \left[-\frac{|\boldsymbol{r}|^{2}}{4 D}\left(\frac{1}{t_{1}^{\prime}}+\frac{1}{t_{2}^{\prime}}+\frac{1}{t_{3}^{\prime}}+\frac{1}{t-t_{3}^{\prime}-t_{2}^{\prime}-t_{1}^{\prime}}\right)\right]\right]
\end{aligned}
$$

However we proceed by Fourier transforming $(47)$ to the $(\boldsymbol{q}, \omega)$-representation to allow direct comparison with the impurity diagram technique. Substituting (36) into (47) and carrying out the integrals over $\boldsymbol{r}, t$ and $t_{1,2,3}^{\prime}$.

$$
\begin{aligned}
R_{3 b}^{\mathrm{sc}}(\omega) \propto \Re \mathrm{e}\left[\left(e^{i \pi / 2}\right)^{3 d / 2-4} \int \mathrm{d}^{d} \boldsymbol{q}_{1} \mathrm{~d}^{d} \boldsymbol{q}_{2} \mathrm{~d}^{d} \boldsymbol{q}_{3}\right. \\
\left.\quad \times\left[\left(\boldsymbol{q}_{1}^{2}+1\right)\left(\boldsymbol{q}_{2}^{2}+1\right)\left(\boldsymbol{q}_{3}^{2}+1\right)\left(\left(\boldsymbol{q}_{1}+\boldsymbol{q}_{2}+\boldsymbol{q}_{3}\right)^{2}+1\right)\right]^{-1}\right]
\end{aligned}
$$

For $d=2$, the integral is convergent, so the term in the square bracket has no real part, and hence $R_{3 b}^{\mathrm{sc}}(\omega)=0$.

Since the semiclassical result for $R_{3 b}^{\mathrm{sc}}$ is zero, the total semiclassical result for the three loop term of the expansion, in a $(2+\epsilon)$-dimensional disordered system without time-reversal symmetry, is given by (46) with $\beta=2$. Expanding the in powers of $\epsilon$, and taking the limit $\epsilon \rightarrow 0$, we neglect the unphysical $1 / \epsilon$ divergent term. Then in 2-dimensions,

$$
R_{3}^{\mathrm{sc}}(s \Delta)=\frac{3}{32 g^{3}} \frac{\ln |s|}{|s|}
$$

where $s=\omega / \Delta$. We now compare this result with the impurity diagram result for $R_{3}(\omega)$, in a system without time reversal symmetry $(\beta=2)$. The geometries of paths that contribute to $R_{3}(\omega)$ are shown in Fig. 1c,d, these can be put in terms of the usual impurity diagrams using Fig. 3. In dimension $d=2+\epsilon$ where $\epsilon$ is small, one finds,

$$
R_{3}(s \Delta)=-\hbar L^{3 d}\left(\frac{\Delta}{2 \pi \hbar}\right)^{4}\left(\frac{1}{3} \epsilon-\frac{1}{2} \epsilon^{2}\right) \Re \mathrm{e}\left[\frac{\partial^{2}}{\partial(i \omega)^{2}}\left[i \omega\left(I_{1}(\omega)\right)^{3}\right]\right]
$$


Using the result for $I_{1}(\omega)$ given in (39), this becomes,

$$
R_{3 a}(s \Delta)=-\frac{1}{32 \pi g^{3}} \frac{1-\frac{9}{4} \epsilon^{2}}{\epsilon} \Re \mathrm{e}\left[(-i s)^{3 \epsilon / 2-1}\right] \stackrel{\epsilon \rightarrow 0}{\longrightarrow}-\frac{3}{128 g^{3}|s|}
$$

This result has a $\left(-|s|^{-1}\right)$ dependence, while the semiclassical result has a $(\ln |s| /|s|)$ dependence. Therefore the semiclassical approach gives a result which is at odds with the impurity diagram result.

We find it instructive to compare the contributions of individual geometries in the two approaches. The physically important quantity is $R_{3}(\omega)$, and one would not necessarily expect $R_{3 a}(\omega)$ and $R_{3 b}(\omega)$ to be the same in the two approaches. However it is of interest to see where the difference between the two approaches comes about. Firstly we will consider the impurity diagram result for $R_{3 a}(\omega)$, this result is the contribution of the path geometry shown in Fig. 2c,

$$
R_{3 a}(\omega)=\frac{8 \hbar L^{3 d}}{3 \beta}\left(\frac{\Delta}{2 \pi \hbar}\right)^{4} \Re \mathrm{e}\left[\frac{\partial^{2}}{\partial(i \omega)^{2}}\left[-\frac{3 i \omega}{2}\left(I_{1}(\omega)\right)^{3}\right]\right]
$$

carrying out one of the differentials over $\omega$,

$R_{3 a}(\omega)=-\frac{4}{\beta} \hbar L^{3 d}\left(\frac{\Delta}{2 \pi \hbar}\right)^{4} \Re \mathrm{e}\left[\frac{\partial}{\partial(i \omega)}\left[\left(I_{1}(\omega)\right)^{3}+\frac{3 i \omega}{\hbar}\left(I_{1}(\omega)\right)^{2} I_{2}(\omega)\right]\right]$

In $(2+\epsilon)$-dimensions where $\epsilon$ is small, this becomes,

$$
R_{3 a}(s \Delta)=\frac{3}{8 \pi \beta g^{3}} \frac{1+\frac{3}{2} \epsilon}{\epsilon^{2}} \Re \mathrm{e}\left[(-i s)^{(3 \epsilon / 2)-1}\right]
$$

Comparing (45) and (54), we see that the impurity diagram approach has an extra term not present in the semiclassical result, just as in the previous order. Like in the two loop case the semiclassics gets the wrong prefactor on the term it does reproduce, in this case a factor of $-3 / 4$. However much worse than this, the second term in (54) has a finite real part in two dimensions, unlike the second term in the two loop diagrammatic result (41). This means the semiclassical result $R_{3 a}^{\mathrm{sc}}$ is very different from the diagrammatic result for $R_{3 a}$.

We now consider the impurity diagram result for $R_{3 b}(\omega)$ in dimension $d=2+\epsilon$, where $\epsilon$ is small. We find,

$$
\begin{aligned}
R_{3 b}(\omega) & =-\frac{2 \hbar L^{3 d}}{\beta}\left(\frac{\Delta}{2 \pi \hbar}\right)^{4}\left(-2+\frac{1}{3} \epsilon-\frac{1}{2} \epsilon^{2}\right) \Re \mathrm{e}\left[\frac{\partial^{2}}{\partial(i \omega)^{2}}\left[i \omega\left(I_{1}(\omega)\right)^{3}\right]\right] \\
& =\frac{3}{16 \beta \pi} \frac{2+\frac{8}{3} \epsilon+\frac{3}{4} \epsilon^{3}}{\epsilon^{2}} \frac{1}{g^{3}} \Re \mathrm{e}\left[(-i s)^{3 \epsilon / 2-1}\right]
\end{aligned}
$$

where $I_{1}(\omega)$ was evaluated using (39), $g$ is the dimensionless conductance given by (33), and $s=\omega / \Delta$. In the limit that $\epsilon \rightarrow 0$ it is clear that (56) is not zero, therefore we find that the semiclassical and impurity diagram technique results are completely different.

The difference between the results of the two approaches can be summarised as follows. In the impurity diagram technique there is no $(\ln |s| /|s|)$ term because the terms in (55) and (56) which are of order $\left(\epsilon^{-2}(-i s)^{3 \epsilon / 2-1}\right)$ exactly cancel each other. In the semiclassics there is no cancellation, and therefore the presence of a prefactor of order $\left(\epsilon^{-2}\right)$ means one must expand $\left((-i s)^{3 \epsilon / 2-1}\right)$ to order $\left(\epsilon^{2}\right)$ to get the $\epsilon$-independent term. It is this that generates the $(\ln |s| /|s|)$ term. 


\section{The weak diagonal expansion for a quantum chaotic system in the ergodic regime.}

It is straightforward to apply the results of the weak diagonal expansion to a system in the ergodic regime. If a system is ergodic, a particle is equally likely to be found anywhere in the system's phase space. This means that the propagation probability for a particle in such a system is featureless and hence the same for all ergodic systems. The method of evaluating the weak diagonal expansion presented here is cast purely in terms of this propagation probability and therefore predicts the same level statistics for all chaotic and disordered systems in the ergodic regime. Given this statement one would like to know two things: (i) are the results of the method discussed in this paper to be believed when applied to the ergodic regime, (ii) are the level statistics predicted by this method the same as those of RMT? A partial answer to the first question would be that it would be difficult to believe the results of the method in the ergodic regime, considering the failure of the method in the diffusive regime of a disordered system. However we can present a better answer to the first question by considering the answer to the second question. While the conjecture that chaotic systems have RMT level statistics [9] is generally considered unproven, this is not the case for disordered systems, for which a proof exists [8]. Therefore if this method's prediction for the ergodic regime of a disordered system does not agree with RMT [10], then this can be considered a proof that the method does not work in the ergodic regime.

To evaluate the weak diagonal approximation results in the ergodic regime, we simply note that in this regime, the average propagation probability, $\left\langle f_{E}\left(\boldsymbol{r}^{\prime}, \boldsymbol{p}^{\prime}, t ; \boldsymbol{r}, \boldsymbol{p}\right)\right\rangle$ is just the inverse volume of the phase space surface with energy, $E$. Thus for a system of volume $L^{d}$, containing semiclassical scatterers, the propagation probability is,

$$
\left\langle f_{E}\left(\boldsymbol{r}^{\prime}, \boldsymbol{p}^{\prime}, t ; \boldsymbol{r}, \boldsymbol{p}\right)\right\rangle=\frac{v_{E}}{L^{d} S_{d} p_{E}^{d-1}}
$$

We can now construct an expansion for the SFF, $K(t)$, in the small parameter $t / t_{\mathrm{H}}$. This expansion can then be compared to the small $t$ expansion of the SFF for random matrices [10]. If each term in the weak diagonal expansion does not agree with the corresponding term in the expansion for random matrices, then we have proven that this method of evaluating the weak diagonal approximation is wrong.

The first term in the SFF is linear in $t / t_{\mathrm{H}}$ and is given by the strong diagonal approximation which agrees with the leading order RMT result [14]. It is well known that for $\beta \neq 2 \mathrm{RMT}$ predicts corrections in powers of $t / t_{\mathrm{H}}$, while for $\beta=2$ the linear in $t$ behaviour is exact for $t<t_{\mathrm{H}}$. The weak diagonal expansion, if correct, must reproduce the higher-order contributions for $\beta=1$ and yet yield no higher-order contributions for $\beta=2$.

In the ergodic regime with time-reversal symmetry $(\beta=1)$ the leading weakdiagonal contribution is the two loop term given by substituting (57) into (23). Comparing this result with the $\beta=1 \mathrm{RMT}$ result, we immediately see it is positive, while the RMT result is negative. Hence we find that this method of evaluating the weak diagonal approximation predicts level statistics different from those of GOE random matrices.

In the $\beta=2$ case, the leading order weak diagonal corrections are given by the three loop terms. It is well known from the impurity diagram technique that in disordered systems such contributions are individually non-zero, but that at each order 
in the perturbation expansion they cancel each other. However when we calculate the three loop terms from the semiclassical approach, we find that both $K_{3 a}$ and $K_{3 b}$ (Fig. $1 \mathrm{c}, \mathrm{d})$ are positive so there is no possibility of them cancelling each other. This means that again this method fails to reproduce the RMT results.

We reiterate that, since we know that disordered systems have random matrix level statistics, the results of this evaluation of the weak diagonal approximation must be wrong.

\section{Concluding remarks.}

The main aim of this paper has been to cast the weak diagonal expansion in terms of semiclassics so that it could be applied to a generic chaotic system. If this could be achieved, the perturbation expansion would be a systematic way of finding corrections to the Berry diagonal approximation; thus in disordered systems such corrections give rise to weak localisation phenomena. If level statistics of chaotic systems are to be RMT, these corrections must be present to describe the difference between GOE statistics and the Berry diagonal approximation for times much shorter than the Heisenberg time.

Having presented this general scheme, we then developed a method of evaluating the terms in the weak diagonal expansion based on the Gutzwiller trace formula. Unfortunately, this method could not correctly reproduce the Hikami boxes which are regions in the phase space where quantum scattering is important. Thus it could neither reproduce the weak localisation expansion for disordered systems, nor give the corrections to the Berry diagonal approximation predicted by RMT. We do not believe that any existing semiclassical approach is capable of correctly evaluating the Hikami boxes for a generic chaotic system. A method exists [6] in which Hikami boxes were calculated for the system of semiclassical scatterers considered here. The problem with this method is that there is no obvious way to generalise it to generic chaotic systems.

Finally we wish to encourage the use of disordered systems as a test bed for theories of quantum chaos. It is one of the few examples of chaotic systems whose quantum behaviour is well understood. In particular, any theory which claims to explain the RMT behaviour of quantum chaotic systems should be tested by checking if it produces the correct level statistics for disordered systems in the diffusive regime. It also appears that it is not enough for a theory to get the correct weak localisation correction to the conductivity of a diffusive system - this is too simple a test to be trusted. A complete semiclassical theory must be able to correctly reproduce higher order corrections to the conductivity and the TLCF.

\section{Acknowledgments}

We are grateful to Oded Agam and Jon Keating for useful discussions. I.V.L thanks the EPSRC for partial support under grant GR/K95505.

[1] Argaman N, Imry Y and Smilansky U 1993 Phys. Rev. B 474440 ; see also Dittrich T and Smilansky U 1991 Nonlinearity 459

[2] Muzykantskii B A and Khmelnitskii D E 1995 JETP Letters 6276

[3] Agam O, Altshuler B L and Andreev A V 1995 Phys. Rev. Lett. 754389

[4] Andreev A V, Agam O, Simons B D and Altshuler B L 1996 Phys. Rev. Lett. 763947 ; 1996 Nucl. Phys. B $\mathbf{4 8 2} 536$ 
[5] Argaman N 1995 Phys. Rev. Lett. 75 2750-2753 ; 1996 Phys. Rev. B 537035

[6] Aleiner I L and Larkin A I 1996 Phys. Rev. B $\mathbf{5 4} 14423$

[7] Cohen D 1998 J. Phys. A 31 277-287

[8] Efetov K B 1982 Sov. Phys. JETP 55514 ; 1997 Sypersymmetry in Disorder and Chaos (Cambridge: Cambridge University Press)

[9] Bohigas O, Giannoni M J and Schmit C 1984 Phys. Rev. Lett. 521

[10] Mehta M L 1991 Random Matrices (San Diego: Academic Press)

[11] Gutzwiller M C 1971 J. Math. Phys. 12343

[12] Altshuler B L and Shklovskii B I 1986 Sov. Phys. JETP 64127

[13] Altland A and Gefen Y 1993 Phys. Rev. Lett. 713339

[14] Berry M V 1985 Proc. R. Soc. London A 400229

[15] Argaman N, Dittes F M, Doron E, Keating J P, Kitaev A Y, Sieber M. and Smilansky U. 1993 Phys. Rev. Lett. $\mathbf{7 1} 4326$

[16] Wegner F J 1979 Z. Phys. B 35207

[17] Gorkov L P, Larkin A I and Khmelnitskii D E 1979 JETP Lett. 30 228; Wegner F and Schäfer L 1980 Z. Phys. B 38 113; McKane A J and Stone M 1981 Ann. Phys. 13136

[18] Zirnbauer M R 1999 in Supersymmetry and Trace Formulae, edited by Lerner I V, Keating J P and Khmelnitskii D E (New York:Plenum) 153

[19] Tripathi V and Khmelnitskii D E 1998 Phys. Rev. B 581122

[20] Blanter Y M, Mirlin A D and Muzykantskii B A 1998 Phys. Rev. Lett. 804161

[21] Abrahams E, Anderson P W, Liccardello D C and Ramakrishnan T V 1979 Phys. Rev. Lett. 42 673

[22] Kravtsov V E and Lerner I V 1995 Phys. Rev. Lett. 742563

[23] Smith R A, Lerner I V and Altshuler B L 1998 Phys. Rev. B 5810343

[24] Hikami S 1981 Phys. Rev. B 242671

[25] Larkin A I and Khemelnitskii D E 1982 Sov. Phys. Usp. 25185 ; Khmelnitskii D E 1984 Physica $B$ \& $C \mathbf{1 2 6} 235$

[26] Bergman G 1984 Phys. Rep. 1071

[27] Chakravarty S and Schmid A 1986 Phys. Rep. 140193

[28] Primack H, Schanz H, Smilansky U and Ussishkin I 1996 Phys. Rev. Lett. 761615 ; 1997 J. Phys. A 306693

[29] Abrikosov A A, Gorkov L P and Dzyaloshinskii I Ye. 1969 Quantum field theoretical methods in statistical physics (New York:Pergamon) 